\title{
Gender dysphoria in children and adolescents - a disease far from pediatric practice?
}

\author{
Mirela Silvia Iancu ${ }^{1,2}$, Ana-Maria Davitoiu ${ }^{1,2}$, Sorina Chindris ${ }^{1,2}$, Iulia Florentina Tincu ${ }^{1,2}$, \\ Monica Livia Gheorghiu ${ }^{1,3}$, Doina Anca Plesca ${ }^{1,2}$ \\ 1"Carol Davila" University of Medicine and Pharmacy, Bucharest, Romania \\ 2"Dr. Victor Gomoiu" Children's Clinical Hospital, Bucharest, Romania \\ 3"C.I.Parhon" National Institute of Endocrinology, Bucharest, Romania
}

\begin{abstract}
Gender dysphoria is not a condition that the Romanian pediatrician commonly encounters in his/her practice. However, in light of the progress made in order to diagnose early and establish a correct treatment, as well as in the evolving context of society, including the one of our country, medical cases will not be long in coming. This paper aims to briefly review, the main characteristics of the disease and the role that the pediatrician can play in the life of the patient already diagnosed or not yet, with gender dysphoria.

Keywords: gender dysphoria, transgene, treatment, child, adolescent
\end{abstract}

\begin{abstract}
Abbreviations
DG - gender dysphoria

DSM IV - Manual of diagnosis and statistics of mental disorders IV

DSM V - Manual of diagnosis and statistics of mental disorders V

WHO - World Health Organization

ICD 11 - International statistical classification of related diseases and health problems

WPATH - World Association of Transgender Health Professionals

GnRH - gonadotropin-releasing hormone
\end{abstract}

\section{INTRODUCTION}

Gender dysphoria (GD) or impairment of gender identity is the discomfort created by the mismatch between the genitals characteristic to certain sex assigned at birth and one's own sense of belonging to a certain gender. This discrepancy can lead to anxiety, restlessness, discomfort, dissatisfaction, suffering and so on, and can negatively influence the quality of life and functionality of the young person in various activities. Hence the need for interventions and treatments that try to synchronize the physical and hormonal profile with what that person thinks and feels.

\section{TERMINOLOGY}

For a better understanding of the different notions used in the text, these are defined below.

Transgender person (or trans person): a person who has a different gender identity from the sex assigned at birth. This notion also includes non-binary people. The term transsexual, which was used in the past, is no longer recommended.

Transgender woman: a person with female gender identity and assigned male sex at birth.

Transgender man: a person with masculine gender identity and assigned female sex at birth. 
Non-binary persons: persons who, regardless of the sex assigned at birth, have a gender identity that is not exclusively male or female, but is a combination of the two (genderqueer, bigender, pangender, genderfluid, or agender).

Cisgen person: the individual whose assigned sex at birth matches (is in accordance to) his or her gender identity.

Gender incongruence: when a person's identity, role or gender expression differs from the cultural norms recommended to a person of a certain sex.

Gender identity: the inner feeling of an individual to feel girl or woman, boy or man, neither, a combination of the two or multiple genders. Hence, it may or may not correspond to the sex assigned to the birth.

Gender expression: the way in which a person presents himself in the outside world as a certain gender, which may not reflect the sex assigned at birth or gender identity and may be different in different environments for the same person, without being a sign of mental disorders. Includes attitude, outfit, clothing, hairstyle, accessories, voice and way of speaking, physical characteristics.

Gender assigned at birth: this is established based on the genitals (male, female or hermaphrodite/intersex), chromosomes and hormones. A distinction must be made between the notions of gender, gender identity and gender expression, as they are distinct entities.

Sexual orientation: the set of emotional, physical, spiritual factors and the gender of people to whom an individual is physically or emotionally attracted. It is completely different from the gender identity and should not be confused with it. Like cis individuals, trans individuals can be heterosexual, gay, lesbian, bisexual, asexual, and the sexual orientation of the latter relates to gender identity.

\section{DEFINITION}

Previously considered gender identity disorder in DSM IV, the condition becomes gender dysphoria in DSM V, and is defined as a severe discordance (incongruence) between the gender experienced or expressed by someone and the gender assigned to that person, manifested for at least 6 months through several aspects.

In children, there is necessary a total of at least 6 of the 8 criteria below:

- The expressed desire to be of the opposite (to the physical one) sex insisting that one is of the opposite sex;

- Preference for the clothing/attire of the other sex (without sexual arousal like the case of transvestite fetishism);
- Persistent preferences for the role of the opposite sex, games of "what would be" or persistent fantasies about having the opposite sex;

- Intense desire to participate in stereotypical games for the opposite sex;

- Preference for playing with those of the opposite sex;

- Intense rejection of typical games/activities for the assigned gender;

- An intense discomfort with one's sexual anatomy;

- A strong desire for primary and/or secondary sexual characters that match the gender he/she lives/experiences.

For adolescents/adults, at least 2 of the 6 criteria below are required:

- Discordance (incongruence) between the gender experienced or expressed by someone and the primary and/or secondary characters (or the anticipated ones, in adolescents);

- Intense desire to get rid of their primary and/or secondary sexual characters due to the discordance between the experienced/ expressed gender and these (in adolescents the desire to prevent the development of the anticipated secondary sexual characters);

- Intense desire to have the primary/secondary sexual characters of the opposite sex;

- Intense desire to be of the opposite sex (or of an alternative sex, different from the assigned one);

- Intense desire to be treated as belonging to the opposite sex (or of an alternative sex, different from the assigned one);

- Believing that they have feelings and reactions typical to the other gender (or to an alternative gender).

The disorder must be associated with significant clinical distress or with the impairment of social, occupational or other type of functionality.

In 2018, the WHO stopped classifying gender incongruity as a mental disorder and in ICD-11, approved in 2019, gender dysphoria is found in the chapter of „Sexual health related disorders“, thus reducing the stigma of these patients and providing the possibility of therapy access.

\section{EPIDEMIOLOGICAL DATA}

There is little data on the actual number of transgender people, much less on those affected by gender dysphoria. This is due to several factors including differences in expression related to the culture of each country, to the stigmatization of these 
patients, which leads to the avoidance of public disclosure, the lack of a clear definition, the fact that among cases with childhood onset many remit until adolescence. According to DSM V, the prevalence of GD is 5-14 per 1,000 adults born boys, and 2-3 for those born girls. The World Professional Association for Transgender Health (WPATH) (1) provides prevalence figures based on studies conducted on patients who address specialized centers, namely between $1: 11,900$ and $1: 45,000$ for trans women and between 1: 30,400 and 1: 200,000 for trans men. In recent years, these figures tend to increase with the increase in the number of people who address a specialist (in the UK, it has doubled during the last 5-6 years (2), while in Toronto the number has increased 4-5 times in 30 years (3)). In addition to the increase in incidence, it seems that the sex ratio is also reversed, in favor of females at birth (4). A questionnaire applied to 2,730 San Francisco children in grades 6-8 showed that $1.3 \%$ identified as trans (5), and a similar questionnaire applied in Minnesota in 2016 to 80,929 students in grades $9-11$ showed a percentage of $3.6 \%$ of those born girls and $1.7 \%$ of those born boys of affirmative answers to the question on whether or not they considered themselves trans or non-binary (6). In Romania, the ACCEPT association estimates that there are at least 60,000 transgender people, whose needs are addressed by the medical system to a small extent.

\section{CLINICAL MANIFESTATIONS}

Gender identity encompasses the psychological aspects of behavior related to femininity or masculinity (Robert Stoller), and DSM V defines it as the selfidentification of an individual as a man or a woman. A child begins to identify as belonging to a gender around the age of 2-3 years, and to this process contributes the "whole" created by the external genitalia and parental and cultural attitudes, which make up cultural gender stereotypes (the perception we form given the cultural influence - school, church, art, press, film) and personal gender stereotypes (our own beliefs with regard to the attributes of genders). At the moment, in most societies, gender is considered binary (male or female) and other variants are vehemently rejected and denied. Meanwhile, a new perspective sees gender as a possible fluid, non-binary, a continuum of possibilities between masculine and feminine extremes, thus accepting various gender identities. Hence the affirmative attitude of psychotherapy recommended by GD specialists, meant to allow and accept the development and exploration of the child's unique way of being, giving him/her the necessary encouragement and help during development.

At a young age, children born boys may be interested in dolls, dresses (cross-dressing appears at the age of 4 in $75 \%$ of those who show it), makeup, women's accessories, games with a female role, while girls can be mainly interested in physical activities, male roles, short haircuts and express their contempt for women's dresses and accessories. As they grow older, they begin to wonder why their bodies look different than they feel and begin to feel anxious that they are not trully perceived by those around them. With the development of the external genitals of the "fake gender", they try to hide them in public. Those with a female phenotype prefer male underwear, refuse to wear feminine swimsuit in public, and hide their breasts by compressing them (breast binding). Meanwhile, those with a male phenotype prefer female underwear, allow their hair to grow long, compress their external genitals (tucking) to have a flat pubic area, urinate from a sitting position. $\mathrm{Pu}-$ berty brings an additional degree of stress with the development of secondary sexual characteristics (especially the beggining of menstruation in those with female phenotype, or erections in those with male phenotype), which confirms the young person that he/she will have to live in a body that does not correspond to their gender identity. Under these circumstances, many young people $(44.3 \%$ of patients in a center specialized in GD) can develop associated psychopathologies: depression, anxiety, bipolar disorder, suicidal thoughts or attempts, self-harming behaviour, eating disorders, pervasive developmental disorder, ADHD, disorder of post-traumatic stress (7, 8). Other problems associated with GD are the bad relationship with parents and their refusal to support them financially, running away from home, stigmatization by colleagues (bullying) and rejection, harassment or physical aggression, sexual abuse, poor school or academic results, substance abuse, risky sexual behavior, with increased chances for HIV infection or other sexually transmitted diseases (9-11).

\section{EVOLUTION}

In evolution, childhood-related GD may or may not continue into adolescence, with the majority usually resolving into adolescence (12). However, severe and persistent forms, as well as forms that worsen with puberty, are likely to be maintained for the long term (13). According to DSM V (14), the rates of GD that persist in adolescence and adulthood vary in the male phenotype between 2 and $30 \%$ and in the female between 12 and $50 \%$. 
The age at which the transgender individual publicly reveals himself to family, friends, schoolmates and others around him/her varies and this is an extremely delicate time for them. Many prefer to do it in writing, with the help of a letter addressed to their parents, thus avoiding the reactions of others and leisurely choosing their words. The Internet is also an environment where transgender young people can "practice" how to reveal themselves, giving them a similar yet virtual world, hence some security and confidence, although it also involves certain risks.

\section{TREATMENT}

The etiology of GD is not known for sure and it is considered to be multifactorial. However, with the development of a database and the increasing number of GD-related studies in children and adolescents, some countries have also created appropriate treatment protocols for this condition. The most widely used are those belonging to the World Professional Association for Transgender Health - WHPAT (1), the Society of Endocrinology (15), the Gender Clinic in Amsterdam (16) and the American Academy of Pediatrics (17). Removing the diagnosis of GD from the Mental Disorders chapter in ICD-11 has also paved the way for an easier medical approach. There are more and more specialized centers worldwide, they have multidisciplinary teams (mental health specialists, endocrinologists, urologists, surgeons, ENT specialists, social workers) trained for approaching patients with GD. These are signs that society is becoming more tolerant and governors accept the allocation of funds for the health of certain medical minorities. At the same time, the addressability and the demand for treatment coming from young transgens increased, yet it is difficult to establish whether it is an increase in the incidence or prevalence of the disease, or an increase in the "visibility" of patients (3).

Treatment for gender dysphoria is divided into psychological therapy and phenotype interventions.

\section{PSYCHOLOGICAL THERAPY}

Each patient must be approached individually in order to find a favorable and appropriate way exploring their gender identity. Currently there are 3 types of psychological approach (18):

1. Affirmative - encourages the exploration of gender identity and supports the young person and family in their going through the desired treatment stages. It represents the therapy recommended by specialists in GD, and it is the one through which the patient is assured that he/she is accepted regardless of the evolution. This therapy includes individual therapy, group therapy for both the young person and for parents, and family therapy.

2. Supportive - applies the principle "wait and see" how gender identity evolves with age and does not advocate for any gender intervention.

3. Corrective - tries to correct and align the gender identity with the patient's biological gender. These have been the most studied methods and are considered inappropriate today because they put the young person under such pressure that can be aggressive or harmful $(1,17)$.

The recommendation for psychological evaluation should be made both to young people with gender dysphoria and to those who, despite not experiencing stress and anxiety related to gender identity, seek to understand and know more details about their situation. Same applies to parents who experience difficult situations related to approving or disapproving their children's identity. It is very important to counsel children who suffer from bullying at school or who have other concomitant psychiatric conditions.

\section{INTERVENTIONS ON THE PHENOTYPE}

Before anything else, patients should be evaluated by both a psychologist and a psychiatrist so as to determine if they meet the criteria necessary in order to be included in the diagnosis of Gender Dysphoria according to DSM V. Subsequently, the family and the young person are prepared for the next steps, they are being explained the benefits and risks of treatment - medicinal or surgical, and the sacrifices involved in assuming the life of the asserted gender identity, in order to limit unrealistic expectations. During this phase, there is also the assessment of possible psychological risk factors (concomitant diseases) and social risk factors (lack of support), all these in case of unfavorable treatment results.

The transition process is gradual, comprising reversible phases (social transition and medication meant to block puberty), partially reversible (hormone therapy of the desired sex), and irreversible (surgery to redefine sex). Not all young trans people want corrective surgery and many give up or stay in one of the stages.

Social transition consists in experiencing life in the desired role (real life). The young person starts living as if he/she belonged to the desired sex by adopting clothes, hairstyle, pronouns, and even another first name, while also hiding the signs of puberty. All of these measures usually alleviate the 
distress of young trans people. The social transition before puberty is debatable and does not require hormonal treatment. There are also young people who want to make the transition only after starting the hormone treatment, since they are fearing the rejection/reactions of those around them.

Hormone treatment to block puberty is indicated for young people whose belief in their gender identity is persistent, consistent and accentuated at puberty, who are relieved of the distress caused by their body developing "wrongly", as well as for those who have not yet defined their beliefs with regard to their own gender identity (non-binary young people), to whom this approach offers additional time to consult with the mental health specialist.

The recommendation is that treatment begin at Tanner 2 stage, which is confirmed by hormonal dosing. It is the stage in which the secondary sexual characters are not sufficiently developed, so that expensive corrective surgeries with questionable results are avoided, yet they do not offer enough material for possible subsequent vulvovaginoplasties. GnRH analogues to gonadectomy (or progestins if there is no GnRH) are used to suppress puberty and to inhibit the hormones of their own gonads. This treatment, known to be effective and safe in early puberty therapy, prevents the appearance of irreversible secondary sexual characteristics (breasts, thickening of the voice, enlargement of the jaw, "Adam's apple", facial hair, too tall stature), has no negative effects on brain development, and is associated with better psychological functionality in adolescents with GD. Of course, the starting this type of treatment is not done without the written consent of the parents. The results reported for this treatment have shown that it is sufficiently safe and has good results when it comes to the patient's satisfaction and psychosocial functionality, but the research is limited (19).

Most patients, however, ask for treatment during advanced stages of puberty, in Tanner stages 3, 4, or 5. In these cases, GnRH analogues are associated with the hormones of the desired gender (cross-gender hormones), helping in lowering their dose and obtaining the necessary level for the desired effect. They have the role of inducing puberty of the desired sex and maintaining an appropriate phenotype. Before starting the cross-gender therapy, the patient should be re-evaluated to see if he/she has psychosocial support, if he/she understands the rational need to follow drug treatment, and the results and risks of treatment. The recommended age for this type of treatment is 16 y.o., the age at which, in some countries, young people have the legal right to make medical decisions. There are also situations in which hor- mone therapy could be started before the age of 16 y.o., in very tall trans young women who need to close the growth cartilages, those with a well-established gender identity before this age, and in order to reduce the trans young people's anxiety caused by comparisons with the somatic pubertal development of their colleagues.

Progressive testosterone administration in trans boys should keep the serum testosterone levels at half the normal ones for men. The treatment causes irreversible changes such as thickening of the voice and enlargement of the clitoris, as well as reversible changes (amenorrhea, moderate increase in facial and body hair depending on the pre-existing distribution, increased muscle mass, redistribution of body fat, increased sexual impulse, sometimes baldness in the temporal region). Possible side effects of this treatment are: thromboembolism, liver dysfunction, hypercholesterolemia, hypertriglyceridemia, endometrial hyperplasia or cancer, which is why hysterectomy is required after the real life stage. The patient has to be warned that, although he is amenorrheic, testosterone does not prevent pregnancy or sexually transmitted diseases, and that for this reason there are required contraception and protective measures. Trans female patients can also benefit from feminization with lower doses of estrogen if they are administered in parallel with $\mathrm{GnRH}$ analogues. Estrogens can cause irreversible breast augmentation, female contours, softer skin, reduced testicles, reduced sexual arousal and calmness. Antiandrogens (spironolactone or cyproterone acetate) may be added if estrogen does not sufficiently lower testosterone or facial hair. The risks of this treatment are high blood pressure, hyperglycemia, liver disorders, thromboembolism, stroke, breast fibroadenoma, prolactinoma, breast and ovarian neoplasms. The risk for cardiovascular mortality has not been proven.

The follow-up of patients with hormone treatment should be done every 3 months in the first year and then, if the evolution is favorable and there are no unwanted effects, at 6 months. Trans boys will be monitored for testosterone levels, serum estradiol levels (compliance indicator), liver tests, lipid profile and serum prolactin. Trans girl patients will be monitored for weight, lipid profile, blood glucose, blood count (for polycythemia), testosterone levels and liver function. Prior to surgery, screening is required for ovarian and uterine cancer in trans men and breast cancer in trans women. Adolescents should be warned that hormonal gender therapy causes infertility in those with suppressed endogenous puberty. In this case, certain methods can be used in order to preserve gametes, but these are expensive and not easy to approach. 
Gender affirmation surgery is performed after the age of 18 y.o., after at least 1 year of hormone treatment and 3 years of psychological and real-life evaluation. The early identification of gender identity issues and the prompt intervention can prevent massive gender confirmation operations. Mastectomy can be performed even in adolescence (20). For trans women, orchiectomy, penectomy, and reconstruction of the vagina and labia majora are performed, and for trans men, hysterectomy with annexectomy and mastectomy, possibly phalloplasty. ENT surgery and phoniatrics are needed to change the voice. After surgery, the doses of hormones are reduced and the decrease in bone density is evaluated at 6-12 months.

\section{CONCLUSIONS}

In conclusion, gender dysphoria is a condition that will be highlighted in the coming years, with

\section{REFERENCES}

1. Standards of Care for the Health of Transsexual, Transgender, and Gender Nonconforming People. The World Professional Association for Transgender Health 7th Version.2012. Available at: https://www. wpath.org/publications/soc.

2. Reed B, Rhodes S, Schofield P, Wylie K. Gender variance in the UK: Prevalence, incidence, growth and geographic distribution. Gender Identity Research and Education Society 2009:1-36.

3. Zucker KJ. Epidemiology of gender dysphoria and transgender identity. Sex Health. 2017;14:404-11.

4. Arnoldusse, M, Steensma TD, Popma A et al. Re-evaluation of the Dutch approach: are recently referred transgender youth different compared to earlier referrals?. Eur Child Adolesc Psychiatry 2020; 29:803-811.

5. Shields JP, Cohen R, Glassman JR et al. Estimating population size and demographic characteristics of lesbian, gay, bisexual, and transgender youth in middle school. J Adolesc Health. 2013; 52:248-50.

6. Rider GN, McMorris BJ, Gower AL et al. Health and Care Utilization of Transgender and Gender Nonconforming Youth: A PopulationBased Study. Pediatrics. 2018;141(3):e20171683.

7. Spack NP, Edwards-Leeper L, Feldman HA et al. Children and Adolescents With Gender Identity Disorder Referred to a Pediatric Medical Center. Pediatrics. 2012;129(3):418-425.

8. Reisner SL, Vetters R, Leclerc M et al. Mental health of transgender youth in care at an adolescent urban community health center: a matched retrospective cohort study. J Adolesc Health. 2015; 56:274-9.

9. Johns MM, Lowry R, Andrzejewski J et al. Transgender Identity and Experiences of Violence Victimization, Substance Use, Suicide Risk, and Sexual Risk Behaviors Among High School Students - 19 States and Large Urban School Districts, 2017. MMWR Morb Mortal Wkly Rep. 2019;68(3):67-71.

10. D'Augelli, A, Grossman A et al. Childhood Gender Atypicality, Victimization, and PTSD Among Lesbian, Gay, and Bisexual Youth. Journal of Interpersonal Violence. 2006;21:1462-82. increased public awareness and availability of information in the media. It is necessary to educate the medical staff on gender identity issues in adolescents. There are already dedicated centers, with programs specifically addressed to gender disorders. Multidisciplinary teams do not include, as standard, a pediatrician, but he may be the first to come into contact with the patient in his practice, to find the disorder and to refer him/her to a mental health specialist for evaluation. It is important that pediatricians are warned with regard to the dynamics of the disease and the patient's particularities such as how to address them, using their preferred first and last name, thus conveying the message that their identity and needs are recognized. Pediatricians can also become advocates for the needs and rights of their trans patients in society.

Conflict of interest: none declared Financial support: none declared

11. Murchison GR, Agénor M, Reisner SL et al. School Restroom and Locker Room Restrictions and Sexual Assault Risk Among Transgender Youth. Pediatrics 2019;143(6):e20182902.

12. Wallien M, Cohen-Kettenis P. Psychosexual Outcome of GenderDysphoric Children. Journal of the American Academy of Child and Adolescent Psychiatry. 2008;47:1413-23.

13. Steensma TD, McGuire JK, Kreukels BPC et al. Factors associated with desistence and persistence of childhood gender dysphoria: A quantitative follow-up study. Journal of the American Academy of Child and Adolescent Psychiatry. 2013;1;52(6):582-590.

14. https://cdn.website-editor.net/30f11123991548a0af708722d458e476/ files/ uploaded/ DSM\%2520V.pdf.

15. Hembree WC, Cohen-Kettenis PT, Gooren L et al. Endocrine Treatment of Gender-Dysphoric/Gender-Incongruent Persons: An Endocrine Society Clinical Practice Guideline. The Journal of Clinical Endocrinology \& Metabolism 2017;102(11):3869-3903.

16. Delemarre-van de Waal HA, Cohen-Kettenis PT. Clinical management of gender identity disorder in adolescents: a protocol on psychological and paediatric endocrinology aspects. Eur $\mathrm{J}$ Endocrinol. 2006;155(suppl 1):S131-S137.

17. Rafferty J, AAP Committee on psychosocial aspects of child and family health, aap committee on adolescence, AAP section on lesbian, gay, bisexual, and transgender health and wellness. Ensuring Comprehensive Care and Support for Transgender and Gender- Diverse Children and Adolescents. Pediatrics. 2018; 142(4): e20182162.

18. Olson J, Forbes C, Belzer M. Management of the transgender adolescent. Arch Pediatr Adolesc Med. 2011;165(2):171-6.

19. Kaltiala-Heino R, Bergman $\mathrm{H}$, Työläjärvi $\mathrm{M}$ et al. Gender dysphoria in adolescence: current perspectives. Adolesc Health Med Ther. 2018; 9:31-41.

20. Handler T, Hojilla JC, Varghese et al. Trends in Referrals to a Pediatric Transgender Clinic. Pediatrics. 2019;144(5):e20191368. 